\title{
Resolvina El promueve resolución de la inflamación en enfermedad periodontal
}

\section{Resolvin El promotes resolution of inflammation in periodontal disease}

\section{ARDILA MEDINA CM*}

\section{RESUIMEN}

Las enfermedades periodontales incluyen procesos inflamatorios en los cuales los factores etiológicos microbiológicos inducen una serie de respuestas del huésped, mediante una cascada de eventos inflamatorios, en un intento por proteger y cicatrizar los tejidos periodontales. Además de las señales celulares y bioquímicas que inician los eventos inflamatorios, las enfermedades periodontales son capaces de generar o detener señales que controlan la inflamación. A parte de los mecanismos tradicionales pro y antiinflamatorios, nuevos mediadores lipídicos endógenos podrían representar una fase de prorresolución. La prorresolución tiene una gran ventaja sobre los métodos tradicionales, debido a que suprime la actividad celular excesiva. A partir de ese concepto, se podría establecer una alternativa de tratamiento empleando formas biológicas de resolución de la inflamación, que limiten la inflamación, y promuevan cicatrización y regeneración con menores riesgos colaterales.

PALABRAS CLAVE: Resolvina El, antiinflamatorio, enfermedad periodontal.

\section{SUMIMARY}

Periodontal diseases are inflammatory processes in which microbial etiologic factors induce a series of host responses that mediate an inflammatory cascade of events in an attempt to protect and heal the periodontal tissues. Beside to the signals that initiate the inflammatory events, periodontal tissues are capable of generating or stop signals that control the inflammation. In addition to the traditional pro- and anti-inflammatory mechanisms, novel endogenous lipid mediators could represent a pro-resolution phase. Pro-resolution has a big advantage over the traditional methods in that it suppresses excessive cellular activity. A new treatment concept where natural pathways of resolution of inflammation can be used to limit inflammation and promote healing and regeneration with minor risk of side-effects.

KEY WORDS: Resolvin El, anti-inflammatory, periodontal disease.

Fecha de recepción: 25 de abril 2009.

Fecha de aceptación: 27 de abril 2009.

\section{INTRODUCCIÓN}

En muchas enfermedades crónicas, la inflamación es el mayor mecanismo de patogénesis de la lesión. La inflamación es una respuesta protectora del huésped a antígenos extraños o a un daño tisular que si no se resuelve, puede conducir a la pérdida de la estructura tisular y la función (1). La inflamación crónica se caracteriza por la producción de citoquinas inflamatorias eicosanoides derivadas del ácido araquidónico (prostaglandinas, tromboxanos, leucotrienos), oxígeno reactivo y moléculas adhesivas (1). En la enfermedad periodontal, como en todas las enfermedades inflamatorias, los eicosanoides derivados del ácido

* Profesor Asistente Facultad de Odontología. Universidad de Antioquía. Miembro Junta Directiva Asociación Colombiana de Periodoncia y Oseointegración. 
araquidónico $(A A)$ juegan un papel clave en la iniciación y patogénesis de la lesión inflamatoria (2). Muchos de los eventos patofisiológicos tempranos de las enfermedades periodontales y su cronicidad pueden ser atribuidos a mediadores lipídicos (3). La resolución de la inflamación es un programa regulado activamente, mejor que la terminación pasiva de la inflamación (4). La crucial identificación de los eventos celulares y señales moleculares que determinan el término de la inflamación y el comienzo de la resolución, conduce a una nueva apreciación de la patogénesis en las enfermedades inflamatorias (5). La evidencia demuestra como mediadores endógenos participan activamente regulando la respuesta del huésped, dirigiendo la resolución de la inflamación (6). La lipoxina, producto de la lipoxigenasa, maneja activamente la resolución de la inflamación (7). Adicionalmente, el papel de circuitos de transformación generados por la aspirina, incluyendo una serie de reacciones celulares y químicas complejas, conduce a una mejor comprensión de la prorresolución (7). Ácidos grasos omega 3 poli-no saturados (AGPS), disminuyen la producción de eicosanoides, citoquinas y oxígeno reactivo, y la expresión de moléculas de adhesión (7). La resolvina El, una nueva molécula que promueve la resolución de la inflamación, es un AGPS metabolizado por la ciclooxigenasa-2 (COX-2) modificada por la aspirina (8). El objetivo de este artículo es presentar la capacidad de la resolvina El para promover la resolución de la inflamación en enfermedad periodontal.

\section{RESOLUCIÓN DE LA INFLAMMACIÓN}

La inflamación aguda tiene diferentes resultados que incluyen su progreso a inflamación crónica, cicatrización y fibrosis o completa resolución (9). La resolución puede ser definida a nivel histológico como el intervalo entre máxima infiltración de neutrófilos y el momento en el que ellos desaparecen de los tejidos (5). Recientemente, ha originado gran interés el mecanismo natural de la resolución, debido al reconocimiento de la inflamación como una característica central en la patogénesis de muchas enfermedades incluyendo aterosclerosis, cáncer, asma, desórdenes neuropatológicos y periodontitis $(10,11)$. La periodontitis es una enfermedad inflamatoria progresiva en la cual los factores etiológicos microbianos inducen una cascada inflamatoria que conduce a la destrucción del periodonto (10). Como en todas las enfermedades inflamatorias, el AA toma parte en el inicio y patogénesis de la lesión inflamatoria en periodontitis, en donde, mediadores lipídicos participan en los eventos pa- tofisiológicos tempranos y en la cronicidad de la enfermedad (11). La resolución de la inflamación es necesaria para el retorno de la enfermedad inflamatoria a un estado saludable, comportándose como un programa regulado activamente (5). Este evento es acompañado por una clase de mediadores lipídicos, que cambian desde prostaglandinas y leucotrienos, a la biosíntesis de mediadores antiinflamatorios como las lipoxinas y nuevas familias de mediadores prorresolución, biosintetizados en exudados de precursores AGPS $(12,13)$. Un AGPS, es metabolizado por COX-2 modificado por la aspirina, para formar una nueva molécula que promueve la resolución de la inflamación: la resolvina El.

\section{NUEVOS MEDIADORES LIPÍDICOS DE LA PRORRESOLUCIÓN INFLAIMATORIA}

Se ha indicado que la generación de lipoxinas (LPXs) estimula la resolución de la inflamación en los tejidos y posiblemente restaura la homeostasis (14). Las LPXs son generadas dentro del lumen vascular por interacciones plaqueto-leucocitarias y biosíntesis transcelular durante respuestas multicelulares como inflamación, aterosclerosis y trombosis (15). La biosíntesis de LPXs se realiza a través de las células mediante lipoxigenasas, interacciones que son reguladas por citoquinas específicas (15). Últimamente, ha despertado gran interés el mecanismo mediante el cual actúa la aspirina en la inflamación. Aunque se conoce su carencia de impacto directo sobre las lipoxigenasas (9), recién se descubrió la tercera vía de biosíntesis de las LPXs, la cual involucra prostaglandina H-II en células endoteliales y 5-lipoxigenasa en leucocitos, generando una nueva 15-epi-lipoxina, cuando la prostaglandina $\mathrm{H}-\mathrm{II}$ se acetila después del tratamiento con aspirina (16). Este nuevo mecanismo de acción para la aspirina demuestra que, además de inhibir mediadores lipídicos como la prostaglandinas, la aspirina posee una habilidad única para provocar la formación de mediadores lipídicos actuando sobre las células endoteliales vasculares (7). Las acciones terapéuticas de la aspirina aún se encuentran en proceso de evolución y continúan sin descubrirse gran cantidad de sus efectos benéficos (17). Se conoce bien la acetilación irreversible de la ciclooxigenasa-1 (COX-1) y (COX-2) con la subsecuente inhibición de prostaglandinas, pero esto no explica todas las acciones farmacológicas de la aspirina (17). Es importante anotar que la biosíntesis de 15-epilipoxina no aparece de una simple maniobra metabólica sino que representa el efecto de la aspirina sobre la función oxigenante de COX-2 (9). Además, la aceti- 
lación de COX-1 mediante aspirina, no permite que cantidades sustanciales de ácido araquidónico se conviertan en 15-epi-lipoxina. Una de las consecuencias desafortunadas del antagonismo de una sola vía enzimática como sucede con los antiinflamatorios no esteroides (AINES) que inhibe específicamente la función COX-2, es la prolongación de la resolución de la inflamación (18). Aun cuando la inhibición de la vía COX-2 usando los AINES, puede atenuar los efectos inflamatorios agudos, la correspondiente reducción en los mediadores lipídicos, tales como prostaglandina $\mathrm{E}_{2}$ $\left(\mathrm{PGE}_{2}\right)$ impide la generación de lipoxinas prorresolución, fundamentales para restaurar la homeostasis tisular (12).

Además de las LPXs derivadas endógenamente o su forma más estable provocada por la aspirina (LPA), se han identificado nuevas clases de mediadores lipídicos de prorresolución (14). Tales moléculas incluyen resolvinas y protectinas derivadas de AGPS, ácido eicosapentanoico (AE) y ácido docosahexanoico (AD) (14). $A E$ se metaboliza a resolvinas de la serie $E(R v E)$ y $A D$ forma resolvinas de la serie D (RvD) y protectinas (14). De una manera similar a las LPXs, protectinas y resolvinas estimulan las vías de antiinflamación y prorresolución, pero lo hacen mediante mecanismos de acción diferentes, adhiriéndose a sitios distintos en las células inflamatorias (14). La administración de aspirina, como sucede con la LPA, incrementa la estabilidad y permanencia de la actividad de las resolvinas y protectinas (9). Las resolvinas estimulan la resolución de la inflamación a través de múltiples mecanismos incluyendo la prevención de penetración de neutrófilos, fagocitando los neutrófilos que sufren apoptosis para clarificar la lesión, e incrementando la solución de la inflamación dentro de la lesión y así promover la regeneración tisular (19). De esta forma, las funciones de prorresolución acortan el intervalo de resolución, ayudando a prevenir la progresión de una respuesta inflamatoria aguda a una inflamación crónica (20).Van Dyke $(14,18)$, empleó un modelo experimental animal para realizar análisis de hibridación ADN-ADN utilizando sondas en microorganismos aislados del periodonto humano, en donde encontró similitud con las especies que colonizan a los conejos. Los resultados de su experimento demostraron que las especies bacterianas encontradas en el periodonto del conejo, previo a la colocación de ligaduras incluían aerobios y anaerobios. La aplicación de Porphyromona gingivalis $(P g)$ a la ligadura, sobre un período de seis semanas seguido por la colocación de un vehículo control por seis semanas adicionales, indujo a la expresión de estos microorganismos en el $90 \%$ de muestras de pla- ca bacteriana examinadas, ocasionando mayor complejidad de la microflora en la biopelícula. Durante este tiempo, ocurrió un cambio dramático en la composición de la placa, volviéndose más patogénica, con una flora predominantemente anaeróbica, caracterizada por la aparición de Aggregatibacter actinomycetemcomitans, además de un incremento de la carga bacteriana. Después de la inducción de la enfermedad, se realizó tratamiento empleando resolvina El (RvEl). Se observó una gran simplificación de la complejidad de la microflora, reduciéndose además la inflamación y destrucción de los tejidos periodontales en este modelo animal.Van Dyke indicó que debido a la ausencia de propiedades antibacterianas directas, son poco claras las razones por las cuales se presentó una reducción de la complejidad de la biopelícula obtenida con RvEl. Se plantearon dos hipótesis: la primera, es que la resolvina promueve la liberación de péptidos antimicrobianos (21), y la segunda es que $P g$ no puede sobrevivir debido a la ausencia de péptidos formados a partir de la degradación del colágeno producida durante la respuesta inflamatoria (18). De esta forma, se sugirió que la magnitud de la inflamación generada por el huésped, determina la composición microbiana en la biopelícula, perpetuando a su vez la respuesta inflamatoria $(14,18)$.

Después de caracterizar el modelo experimental de enfermedad periodontal inducido por $P g$, se exploró la posibilidad de prevenir la inflamación y la pérdida ósea, mediante la aplicación tópica de RvEl, colocando ligaduras alrededor de los segundos premolares inferiores de conejos durante seis semanas, creando un ambiente para el crecimiento de $\mathrm{Pg}$ (adicionado exógenamente) (20). Se ligó otro grupo de animales $\sin P g$ y un tercer grupo se manejó sin ligadura ni $P g$. Con el propósito de una comparación directa, a un cuarto grupo se le administró metronidazol sistémicamente con el fin de eliminar Pg, y así reducir la inflamación y el daño tisular, proporcionando medios directos que permitieran evaluar la terapia sobre el huésped, a través del uso del mediador RvEl versus metronidazol. La aplicación de $P g$ junto con las ligaduras condujo al desarrollo de periodontitis. Se inhibió el inicio de la enfermedad mediante $P g$ aplicando $50 \mathrm{mg}$ IM de metronidazol. El tratamiento tópico con RvEl, tres veces por semana en el sitio de la ligadura, inhibió el daño tisular y óseo > 95\%. RvEl no presentó actividad antibacteriana intrínseca y no impidió el crecimiento de Pg. Los conejos toleraron adecuadamente el procedimiento y no presentaron eventos adversos. Mediante evaluación radiográfica, se determinó que RvEl presentó una protección cuatro 
veces mayor de la pérdida ósea cuando se comparó con los otros grupos. En los animales no tratados con RvEl, el análisis histológico demostró prominente infiltrado leucocitario y pérdida ósea, mientras que en los tratados con RvEl no se observaron neutrófilos ni daño tisular.

Conocida la capacidad de RvEl para prevenir el establecimiento y progreso de la periodontitis, Hasturk y colaboradores (22), evaluaron el potencial de REl como molécula prorresolución inflamatoria para el tratamiento de periodontitis establecida. Se empleó un sistema in vivo para evaluar la destrucción de los tejidos duros y blandos en periodontitis experimental. Nuevamente, se usó Pg para inducir la periodontitis en 39 conejos neozelandeses. Para determinar el estado de la enfermedad en la línea de base, se sacrificó un grupo a la sexta semana. Los otros grupos ingresaron al tratamiento durante seis semanas adicionales. Los brazos de tratamiento del estudio incluyeron RvEl como monoterapia; como grupo placebo control se utilizó $95 \%$ de etanol; se emplearon como monoterapia alternativa $\mathrm{PGE}_{2}$ y leucotrieno $\mathrm{B}_{4}\left(\mathrm{LTB}_{4}\right)$. Los autores concluyeron que la terapia con placebo no afectó el progreso de la enfermedad, el cual fue significativamente más agresivo cuando se utilizó $\mathrm{PGE}_{2}$ o $\mathrm{LTB}_{4}$. El tratamiento tópico con RvEl ocasionó una resolución completa de la inflamación periodontal y restablecimiento clínicamente visible de los tejidos blandos y duros. También se resolvió la apariencia irregular, edematosa e hiperémica de los tejidos blandos y se produjo un crecimiento óseo mayor a los niveles presentados en la enfermedad de base. Adicionalmente se indicó que la enfermedad periodontal progresó en cada uno de los grupos restantes con relación a su estado inicial.

\section{CONCLUSIONES}

La evidencia actual, basada en modelos experimentales en fase preclínica, demuestra que la resolvina El actúa como modulador de la respuesta inflamatoria, transformándola a una resolución más rápida, previniendo efectivamente la fase crónica. La eliminación de la inflamación en la lesión cicatrizada promueve la regeneración tisular, incluyendo tejido conectivo y hueso, ofreciendo una ventaja terapéutica en el tratamiento de la periodontitis cuando se compara con las alternativas farmacológicas tradicionales. Aunque el potencial proinflamatorio de la RvEl es inmenso, su aplicación en seres humanos tardará un poco debido a que se tienen que realizar estudios clínicos que cum- plan las fases I a IV para así determinar su eficacia a largo plazo.

\section{BIBLIOGRAFÍA}

1. Medzhitov R. Origin and physiological roles of inflammation. Nature 2008;24:428-35.

2. Silva N, Dutzan N, Hernandez M, Dezerega A, Rivera O, Aguillon JC, Characterization of progressive periodontal lesions in chronic periodontitis patients: levels of chemokines, cytokines, matrix metalloproteinase-13, periodontal pathogens and inflammatory cells. J Clin Periodontol 2008;35:206-14.

3. Offenbacher S, Barros SP, Beck JD. Rethinking periodontal inflammation. J Periodontol 2008;79 (8 Suppl):157784.

4. Serhan CN, Savill J. Resolution of inflammation: the beginning programs the end. Nat Immunol 2005;6:1 1917.

5. Serhan, CN, Brain SD, Buckley CD, Gilroy DW, Haslett C, O'Neill LA, et al. Resolution of inflammation: state of the art, definitions and terms. FASEB J 2007;21:325-32.

6. Hong S, Gronert K, Devchand PR, Moussignac RL, Serhan CB. Novel docosatrienes and 17S-resolvins generated from docosahexaenoic acid in murine brain, human blood, and glial cells: autacoids in anti-inflammation. J Biol Chem 2003;278:14677-87.

7. Serhan CN, Arita M, Hong S, Gotlinger K. Resolvins, docosatrienes, and neuroprotectins, novel omega-3derived mediators, and their endogenous aspirintriggered epimers. Lipids 2004;39:1125-32.

8. Serhan CN, Gotlinger K, Hong S, Arita M. Resolvins, docosatrienes, and neuroprotectins, novel omega-3derived mediators, and their aspirintriggered endogenous epimers: an overview of their protective roles in catabasis. Prostaglandins Other Lipid Mediat 2004;73:155-72.

9. Serhan CN, Chiang N, Van Dyke TE. Resolving inflammation: dual anti-inflammatory and pro-resolution lipid mediators. Nat Rev Immunol 2008;8:349-61.

10. Hansson GK, Robertson AKL, Soderberg-Naucler C. Inflammation and atherosclerosis. Annu Rev Pathol Mech Dis 2006;1:297-329. 
10. Kinane DF, Lappin DF. Immune processes in periodontal disease: a review. Ann. Periodontol 2002;7:62-71.

11. Okada H, Murakami S. Cytokine expression in periodontal health and disease. Crit Rev Oral Biol Med 1998; 9:248-66.

12. Levy BD, Clish CB, Schmidt B, Gronert K, Serhan CN. Lipid mediator class switching during acute inflammation: signals in resolution. Nature Immunol 2001;2: 612-19.

13. Hong S, Gronert K, Devchand P, Moussignac R-L, Serhan CN. Novel docosatrienes and 17S-resolvins generated from docosahexaenoic acid in murine brain, human blood and glial cells: autacoids in anti-inflammation. J Biol Chem 2003;278:14677-87.

14. Van Dyke TE. Control of inflammation and periodontitis. Periodontol 2000 2007;45:158-66.

15. Serhan CN, Chiang N. Endogenous pro-resolving and anti-inflammatory lipid mediators: a new pharmacologic genus. Br J Pharmacol 2008;153 Suppl 1:S20015.

16. Clària J, Serhan CN. Aspirin triggers previously undescribed bioactive eicosanoids by human endothelial cellleukocyte interactions. Proc Natl Acad Sci USA 1995;92:9475-9.

17. Ridker PM, Cook NR, Lee IM, Gordon D, Gaziano JM, Manson JE, et al. A randomized trial of low-dose aspirin in the primary prevention of cardiovascular disease in women. N Engl J Med 2005;352:1293-304.

18. Van Dyke TE. The management of inflammation in periodontal disease. J Periodontol 2008;79(8 Suppl):1601-8.

19. Schwab JM, Chiang N, Arita M, Serhan CN. Resolvin El and protectin Dl activate inflammation-resolution programmes. Nature 2007;447:869-74.

20. Hasturk H, Kantarci A, Goguet-Surmenian E, Blackwood A, Andry C, Serhan CN, et al. Resolvin El regulates inflammation at the cellular and tissue level and restores tissue homeostasis in vivo. J Immunol 2007;179:7021-9.

21. Levy O, Canny G, Serhan CN, Colgan SP. Expression of BPI (bactericidal/permeability-increasing protein) in human mucosal epithelia. Biochem Soc Trans 2003;31: 795-800.

22. Hasturk H, Kantarci A, Ohira T, Arita M, Ebrahimi N, Chiang $\mathrm{N}$, et al. RvEl protects from local inflammation and osteoclast- mediated bone destruction in periodontitis. FASEB J 2006;20:401-3.

\section{CORRESPONDENCIA}

Carlos Martín Ardila Medina

Carrera 47 No. 20 sur 46

Envigado Antioquía Colombia

57(4) 3348122

martinardila@gmail.com 